\title{
Performance of a Shock Tube with a Large-Area Contraction
}

\author{
George Emanuel, ${ }^{*}$ U. S. Satyanand,$^{\dagger}$ and Frank K. Lu ${ }^{\ddagger}$ \\ University of Texas at Arlington, Arlington, Texas 76019
}

\begin{abstract}
An inviscid, analytical model is provided for shocktube performance when there is a large, abrupt contraction in the low-pressure side of a tube. The analysis is independent of initial high-pressure side conditions. Only the ratio of specific heats of the low-pressure gas, the large-diameter tube's incident shock Mach number, and the contraction ratio are needed for a nondimensional solution of the transmitted flow in the small-diameter tube. This solution is unique, readily evaluated, and various limits and constraints are discussed. Parametric calculations establish the increased magnitude of the transmitted shock Mach number relative to the shock Mach number for a tube without contraction. The Mach number increase, then increases the pressure and temperature downstream of the small-diameter tube's incident and reflected shocks, again relative to a tube without a contraction. Shock-tube performance, therefore, can be improved by the addition of a small-diameter tube to an existing tube's low-pressure side. The improvement can be used with a combustion-driven tube, a free-piston tube, etc.
\end{abstract}

\section{Nomenclature}

\section{A}

$a$

$J_{+}$

$M$

$p$

$R$

$\bar{R}$

$s$

$T$

$W$

$w$

$X$

Y

Z

$\alpha$

$\gamma$

$\rho$

Subscripts

$L$

$R$

$r$

$S$

$t$

0

$1,2, \ldots, 10$

Superscript

(1)

$=$ first estimate

\section{Introduction}

T HE low-pressure side of a shock tube is sometimes modified from its usual closed-end configuration. Modifications include

Received 11 August 2004; revision received 13 April 2005; accepted for publication 21 April 2005. Copyright (C) 2005 by the American Institute of Aeronautics and Astronautics, Inc. All rights reserved. Copies of this paper may be made for personal or internal use, on condition that the copier pay the $\$ 10.00$ per-copy fee to the Copyright Clearance Center, Inc., 222 Rosewood Drive, Danvers, MA 01923; include the code 0001-1452/05 \$10.00 in correspondence with the CCC.

*Professor, Department of Mechanical and Aerospace Engineering. Associate Fellow AIAA.

$\dagger$ Graduate Research Associate, Department of Mechanical and Aerospace Engineering.

Professor and Director, Aerodynamics Research Center, Department of Mechanical and Aerospace Engineering. Associate Fellow AIAA. a Ludwieg tube $e^{1,2}$ or a shock tunnel. ${ }^{3}$ Another modification is an abrupt, large contraction in the low-pressure side that leads into a small-diameter tube with a closed end. Adding a small-diameter tube to an existing facility is simple and cost effective. Moreover, it does not conflict, for example, with free-piston or combustiondriven operation. A sudden contraction increases the transmitted shock Mach number $M_{t}$ relative to the large-diameter tube's incident shock Mach number $M_{s}$. In turn, this increases the pressure, temperature, and Mach number, $M_{8}$, in the gas downstream of the transmitted shock. Moreover, the pressure and temperature behind the reflected shock in a small-diameter tube can significantly exceed that behind the reflected shock in the closed end of a largediameter tube. In addition to the preceding, a variety of approaches have been used to improve the performance of shock tubes. ${ }^{4}$ These include increasing the pressure or temperature of the driver gas, using a light driver gas, or using expansion, free-piston, combustion or detonation drivers. ${ }^{3-17} \mathrm{~A}$ further increase in performance can be achieved by combining these techniques with an area contraction.

The facilities just highlighted, however, were without the benefit of a contraction to further increase the temperature in the reflected shock gas region. In general, the temperature, or enthalpy, in a smalldiameter tube's reflected shock region can exceed by about $50 \%$ that in a large-diameter tube's reflected shock region. A contraction is thus beneficial for radiative heat transfer, chemical kinetic, and plasma studies, as well as for shock-tunnel operation. Moreover, it can be used in combination with other performance enhancing techniques, such as a light driver gas.

A sudden or a gradual contraction of the tube at the diaphragm section also increases the incident shock Mach number. Alpher and White ${ }^{18}$ provide an inviscid analysis for a gradual monotonic convergence of the tube in the diaphragm section. Nonideal diaphragm rupture, however, complicates flow behavior and results in a different and more complex model than the one discussed here. Operationally, this approach is more involved than a low-pressure side contraction.

Salas ${ }^{19}$ provides an analysis for an abrupt area change, either expanding or contracting, away from the diaphragm and in the lowpressure side of the tube. The analysis, however, is limited to a small area change. In this circumstance, a multiplicity of solutions is possible, and the computational procedure utilizes an involved iteration. Both the Alpher and White ${ }^{18}$ and Salas ${ }^{19}$ analyses are one dimensional, that is, they model flow conditions along the axis of the tube, as is the case in the present study. By limiting the area contraction to a relatively large value, say two or more, a unique solution is obtained. The iterative procedure of Salas ${ }^{19}$ is also removed.

Standard assumptions are utilized for the inviscid analysis of an idealized shock-tube flow. Perfect, possibly different, gases are used in the high- and low-pressure sections. The analysis for incident flow conditions in the large-diameter tube is well known ${ }^{20}$ and is 


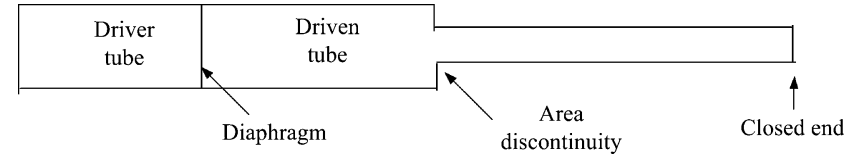

a)

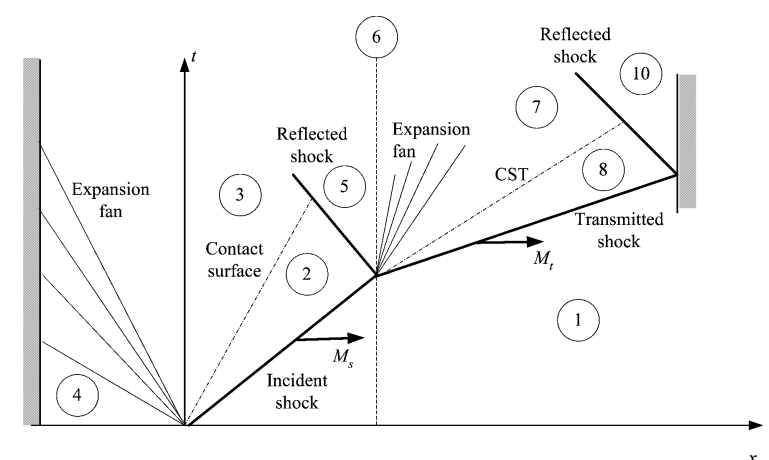

b)

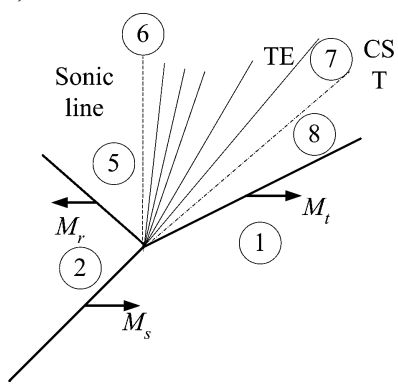

5

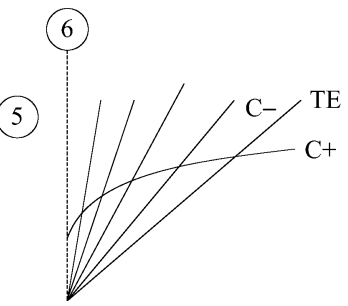

c)

d)

Fig. 1 Configuration and wave diagram for a shock tube with a largearea contraction in the driven section: a) schematic, b) idealized wave diagram, c) wave diagram in the vicinity of the contraction, and d) wave diagram for the rarefaction.

not reproduced. The temperature $T_{1}$, pressure $p_{1}$, molecular weight $W$, and ratio of specific heats $\gamma$ of the gas in the low-pressure side of the tube are presumed known, as is the Mach number $M_{s}$ for the incident shock in the large-diameter tube (see Fig. 1). As a consequence, values for $\gamma_{4}, W_{4}, T_{4}$, and $p_{4}$ for the high-pressure gas are unnecessary. The subsequent analysis thus terminates before flow conditions, associated with the high-pressure gas, alter the flow associated with the contraction. Along with $T_{1}, p_{1}, \gamma, W$, and $M_{s}$, the only other specified parameter is the contraction area ratio $\alpha$.

Pressure, temperature, and density are normalized by their state 1 values, and flow speeds are normalized by the speed of sound in state $1, a_{1}$. A nondimensional solution is only a function of $\gamma, M_{s}$, and $\alpha$. For instance, these parameters are sufficient to establish the transmitted shock Mach number $M_{t}$. Relations are provided for flow conditions of the gas between the transmitted shock and the contact surface in the small-diameter tube and for conditions behind the reflected shock. This surface stems from the wave reflection process that starts at the contraction. Results are developed under the assumption of a large contraction ratio, that is, $\alpha \gg 1$. Later analysis shows that there is relatively little benefit associated with a contraction when $\alpha$ is in excess of about 5 .

A novel analytical model is presented in the next section. The last section outlines parametric calculations and briefly summarizes results.

\section{Analysis}

\section{Preliminary Remarks}

The overall model is described before key features are discussed. Figure 1 contains a schematic and wave diagrams for idealized shock-tube flow with an abrupt, large contraction in the driven section. Regions 1 and 4 contain the initial low- and high-pressure gases in the driven and driver sections, respectively. To the left of state 6, the cross-sectional area is $A_{L}$, and to the right it is $A_{R}$, where $\alpha=\left(A_{L} / A_{R}\right)$. The incident shock partly reflects from the contraction thereby giving rise to region 5. Part of the incident shock propagates into the small diameter tube with a Mach number $M_{t}$. Region 8 is a uniform flow region between the transmitted shock and the contact surface, labeled CST. Pressure and velocity tangency conditions apply across CST. Region 7 is also a uniform flow region. A rarefaction wave is required between states 6 and 7, where state 6 is sonic. This description is based on Salas, ${ }^{18}$ who validates it using a quasi-one-dimensional time-dependent Euler code.

Figure 1c provides an enlargement of the flowfield in the vicinity of the contraction. In a closed-end shock tube, region 5 is quiescent. This is also the case for the gas at the outer tube wall, away from the opening. Gas, however, flows through the inlet of the small diameter tube. Consequently, the gas is not quiescent on the tube's axis, and $M_{5}$ has a small positive value, which stems from a positive flow speed $w_{5}$ just downstream of the inlet to the small-diameter tube. The reflected shock is thus strongest at its perimeter and slightly weaker on the tube's axis. The reflected shock, in the figure, is for the shock on the axis, where its Mach number is $M_{r}$.

The combination of incident and reflected shocks in the largediameter tube is stronger than the transmitted shock. Hence, the pressure and specific entropy satisfy

$$
p_{5}>p_{8}, \quad s_{5}>s_{8}
$$

The pressure inequality requires a rarefaction wave whose sonic leading edge is at state 6 . The strength of the rarefaction is such that the pressure at its trailing edge (TE) matches the pressure just downstream of the transmitted shock. Because the entropy in regions 5 , 6,7 and throughout the rarefaction wave is the same, the entropy in region 7 exceeds that in region 8 . These two regions are therefore separated by a contact surface labeled CST.

There is a limitation on the maximum strength of the transmitted shock because $p_{8}$ cannot exceed $p_{5}$. On the other hand, the $M_{s}$ Mach number represents a lower bound for $M_{t}$ because the transmitted shock cannot have this value, as it corresponds to a quiescent gas, on the axis, in region 5 . The model thus bounds $M_{t}$, where the lower bound is $M_{s}$.

Validity of the model is based on Euler code ${ }^{18}$ results. A selfconsistent unique solution is obtained, and second-law violations are avoided. This means that the following entropy inequalities apply:

$$
s_{1}<s_{2}<s_{5}, \quad s_{1}<s_{8}<s_{5}
$$

\section{Region 5}

Conservation of mass for a steady flow across the contraction yields

$$
\alpha=A_{L} / A_{R}=\left(M_{6} / M_{5}\right)\left(X_{5} / X_{6}\right)^{(\gamma+1) /[2(\gamma-1)]}
$$

where the $X, Y$, and $Z$ Mach-number functions are defined in the Nomenclature. With $M_{6}=1$, this simplifies to

$$
\alpha=\left(1 / M_{5}\right)\left\{[2 /(\gamma+1)] X_{5}\right\}^{(\gamma+1) /[2(\gamma-1)]}
$$

Because $\alpha$ is prescribed, solve for $M_{5}$ to obtain

$$
[(\gamma+1) / 2]\left(\alpha M_{5}\right)^{2(\gamma-1) /(\gamma+1)}-[(\gamma-1) / 2] M_{5}^{2}-1=0
$$

When $\alpha=1$, there is no contraction, regions 5-7 do not exist, region 8 is identical to region 2, and $M_{t}=M_{s}$. Equation (3), however, requires $M_{6}=1$ and yields $M_{5}=1$ when $\alpha=1$. The model represents a different flowfield, compared to that with no contraction, and is therefore singular in the $\alpha=1$ limit. At the other extreme, when $\alpha \gg>1, M_{5}$ is nearly zero, and a first estimate is obtained by disregarding the $M_{5}^{2}$ term in Eq. (3):

$$
M_{5}^{(1)}=[2 /(\gamma+1)]^{(\gamma+1) /[2(\gamma-1)]}(1 / \alpha)
$$

This estimate differs from $M_{5}$ of Eq. (3) by less than $2.3 \%$ when $\alpha=3$ and $\gamma=1.4$. The difference further decreases as $\alpha$ increases. 
The $\alpha \rightarrow \infty$ case results in a quiescent gas in region 5 , which is also a singular limit for the model.

For a reflected shock, $M_{r}$ and $\tilde{M}_{r}$ here denote a shock-fixed Mach number and a laboratory Mach number, respectively. Emanuel ${ }^{21}$ [Eq. (12.25b)] provides a relation for a reflected shock in which the downstream flow is not necessarily quiescent. With the current notation, this relation is

$M_{5}=\frac{1-[(\gamma+1) / 2] \tilde{M}_{r}\left(M_{2}+\tilde{M}_{r}\right)+[(\gamma-1) / 2]\left(M_{2}+\tilde{M}_{r}\right)^{2}}{\left(\left\{1+[(\gamma-1) / 2]\left(M_{2}+\tilde{M}_{r}\right)^{2}\right\}\left[\gamma\left(M_{2}+\tilde{M}_{r}\right)^{2}-(\gamma+1) / 2\right]\right)^{\frac{1}{2}}}$

The single unknown is $\tilde{M}_{r}$ because $M_{2}$ is evaluated in the appendix and $M_{5}$ by Eq. (3). A first $\tilde{M}_{r}^{(1)}$ estimate is obtained by setting the numerator equal to zero:

$$
\tilde{M}_{r}^{(1)}=\left\{1+[(\gamma+1) / 4]^{2} M_{2}^{2}\right\}^{\frac{1}{2}}-[(3-\gamma) / 4] M_{2}
$$

Starting with this value, $\tilde{M}_{r}$ is numerically established. The shockfixed Mach number

$$
M_{r}=M_{2}+\tilde{M}_{r}
$$

is used to establish the following region 5 properties:

$$
\begin{gathered}
p_{5} / p_{1}=[2 /(\gamma+1)] Y\left(M_{r}\right)\left(p_{2} / p_{1}\right) \\
T_{5} / T_{1}=[2 /(\gamma+1)]^{2}\left[X\left(M_{r}\right) Y\left(M_{r}\right) / M_{r}^{2}\right]\left(T_{2} / T_{1}\right) \\
a_{5} / a_{1}=\left(T_{5} / T_{1}\right)^{\frac{1}{2}}
\end{gathered}
$$

where $p_{2} / p_{1}$ and $T_{2} / T_{1}$ are given in the Appendix.

\section{State 6}

The speed of sound at state 6 is established by the conditions

$$
\begin{gathered}
s_{6}=s_{5} \\
M_{6}=1 \\
T_{06}=T_{05}
\end{gathered}
$$

where $T_{0}$ is the stagnation temperature. Observe that the stagnation temperature relation is equivalent to an energy equation, and this means that the flow between regions 5 and 6 is viewed as steady. Equations (9a) and (9c), respectively, yield

$$
\begin{gathered}
p_{6} / p_{5}=\left(T_{6} / T_{5}\right)^{\gamma /(\gamma-1)} \\
T_{6} / T_{5}=X_{5} / X_{6}
\end{gathered}
$$

With Eq. (9b), the preceding reduces to

$$
a_{6} / a_{1}=\left\{[2 /(\gamma+1)] X_{5}\right\}^{\frac{1}{2}}\left(a_{5} / a_{1}\right)
$$

where $M_{5}$ and $a_{5} / a_{1}$ are given, respectively, by Eqs. (3) and (8c).

\section{State 7}

Conditions associated with state 7 require the theory of characteristics for a simple, centered rarefaction wave. ${ }^{21}$ Figure $1 \mathrm{~d}$ is a sketch of the rarefaction, where the $C_{-}$characteristics are straight, while curved $C_{+}$characteristics connect states 6 and 7 . The relevant equations are

$$
\begin{gathered}
s_{7}=s_{6} \\
J_{+6}=J_{+5}
\end{gathered}
$$

where $J_{+}$is a Riemann invariant:

$$
J_{+}=w+[2 /(\gamma-1)] a
$$

Equations (13b) and (14) yield

$$
a_{7}\left\{1+[(\gamma-1) / 2] M_{7}\right\}=[(\gamma+1) / 2] a_{6}
$$

and Eqs. (9a) and (13a) provide

$$
p_{7} / p_{5}=\left(T_{7} / T_{5}\right)^{\gamma /(\gamma-1)}=\left(a_{7} / a_{5}\right)^{2 \gamma /(\gamma-1)}
$$

\section{$M_{t}$ and $a_{7} / a_{1}$}

The CST surface requires

$$
\begin{gathered}
p_{8}=p_{7} \\
w_{8}=w_{7}
\end{gathered}
$$

The incident shock in the small-diameter tube yields

$$
p_{8} / p_{1}=[2 /(\gamma+1)] Y_{t}
$$

or

$$
M_{t}=\left((1 / \gamma)\left\{[(\gamma+1) / 2]\left(p_{8} / p_{1}\right)+(\gamma-1) / 2\right\}\right)^{\frac{1}{2}}
$$

With the aid of Eqs. (16) and (17a), $p_{8} / p_{1}$ can be eliminated with

$$
p_{8} / p_{1}=\left(p_{7} / p_{5}\right)\left(p_{5} / p_{1}\right)=\left(a_{7} / a_{5}\right)^{2 \gamma /(\gamma-1)}\left(p_{5} / p_{1}\right)
$$

where $p_{5} / p_{1}$ is given by Eq. (8a). The preceding two equations combine to yield

$$
\gamma M_{t}^{2}-\frac{\gamma+1}{2} \frac{p_{5} / p_{1}}{\left(a_{5} / a_{1}\right)^{2 \gamma /(\gamma-1)}}\left(\frac{a_{7}}{a_{1}}\right)^{2 \gamma /(\gamma-1)}=\frac{\gamma-1}{2}
$$

whose unknowns are $M_{t}$ and $a_{7} / a_{1}$.

A second equation for $M_{t}$ and $a_{7} / a_{1}$ is developed starting from Eq. (15). First, $M_{7}$ is eliminated with

$$
\begin{gathered}
w_{8} / a_{1}=[2 /(\gamma+1)]\left[Z\left(M_{t}\right) / M_{t}\right]=w_{7} / a_{1} \\
M_{7}=\left(w_{7} / a_{1}\right)\left(a_{1} / a_{7}\right)=[2 /(\gamma+1)]\left[Z\left(M_{t}\right) / M_{t}\right]\left(a_{1} / a_{7}\right)
\end{gathered}
$$

Equation (15) thus becomes

$$
a_{7} / a_{1}+[(\gamma-1) /(\gamma+1)]\left[Z\left(M_{t}\right) / M_{t}\right]=[(\gamma+1) / 2]\left(a_{6} / a_{1}\right)
$$

where $a_{6} / a_{1}$ is given by Eq. (12). Equations (20) and (23) are two coupled equations for $M_{t}$ and $a_{7} / a_{1}$, where

$$
1<M_{s}<M_{t}
$$

The $a_{7} / a_{1}$ parameter can be eliminated, but this was not done because the coupled equations are readily solved numerically. Once $M_{t}$ and $a_{7} / a_{1}$ are established, $M_{7}$ is given by Eq. (22).

\section{Region 8}

The state of the gas in region 8 is given by

$$
\begin{gathered}
p_{8} / p_{1}=[2 /(\gamma+1)] Y_{t} \\
T_{8} / T_{1}=[2 /(\gamma+1)]^{2}\left(X_{t} Y_{t} / M_{t}^{2}\right) \\
\rho_{1}=p_{1} / R T_{1} \\
\rho_{8} / \rho_{1}=[(\gamma+1) / 2]\left(M_{t}^{2} / X_{t}\right) \\
M_{8}=Z_{t} /\left(X_{t} Y_{t}\right)^{\frac{1}{2}} \\
a_{8} / a_{1}=\left(T_{8} / T_{1}\right)^{\frac{1}{2}} \\
w_{8} / a_{1}=\left(a_{8} / a_{1}\right) M_{8} \\
w_{t} / a_{1}=M_{t}
\end{gathered}
$$


Table 1 Parametric $M_{s}$ results when $\gamma=1.4$ and $\alpha=5$

\begin{tabular}{|c|c|c|c|c|c|c|c|c|c|c|c|c|}
\hline \multirow[b]{2}{*}{$M_{s}$} & \multirow[b]{2}{*}{$M_{t}$} & \multirow[b]{2}{*}{$M_{2}$} & \multirow[b]{2}{*}{$M_{8}$} & \multirow[b]{2}{*}{$p_{2} / p_{1}$} & \multirow[b]{2}{*}{$p_{8} / p_{1}$} & \multirow[b]{2}{*}{$T_{2} / T_{1}$} & \multirow[b]{2}{*}{$T_{8} / T_{1}$} & \multirow[b]{2}{*}{$w_{2} / a_{1}$} & \multirow[b]{2}{*}{$w_{8} / a_{1}$} & \multicolumn{3}{|c|}{$p_{4} / p_{1}$} \\
\hline & & & & & & & & & & Air/air & Helium/air & Helium/argon \\
\hline 2.5 & 2.891 & 1.197 & 1.327 & 7.130 & 9.580 & 2.138 & 2.553 & 1.750 & 2.121 & 145.3 & 21.56 & 18.75 \\
\hline 3.0 & 3.478 & 1.358 & 1.467 & 10.33 & 13.94 & 2.679 & 3.284 & 2.222 & 2.658 & 632.6 & 44.21 & 35.93 \\
\hline 3.5 & 4.065 & 1.471 & 1.562 & 14.13 & 19.11 & 3.315 & 4.149 & 2.679 & 3.183 & 3037 & 86.53 & 65.24 \\
\hline 4.0 & 4.653 & 1.553 & 1.630 & 18.50 & 25.09 & 4.047 & 5.148 & 3.125 & 3.699 & $1.774 E+4$ & 165.4 & 114.6 \\
\hline 4.5 & 5.242 & 1.615 & 1.679 & 23.46 & 31.89 & 4.875 & 6.281 & 3.565 & 4.209 & $1.461 E+5$ & 313.7 & 197.4 \\
\hline 5.0 & 5.830 & 1.661 & 1.716 & 29.00 & 39.48 & 5.800 & 7.549 & 4.000 & 4.715 & $2.266 E+6$ & 598.1 & 337.0 \\
\hline
\end{tabular}

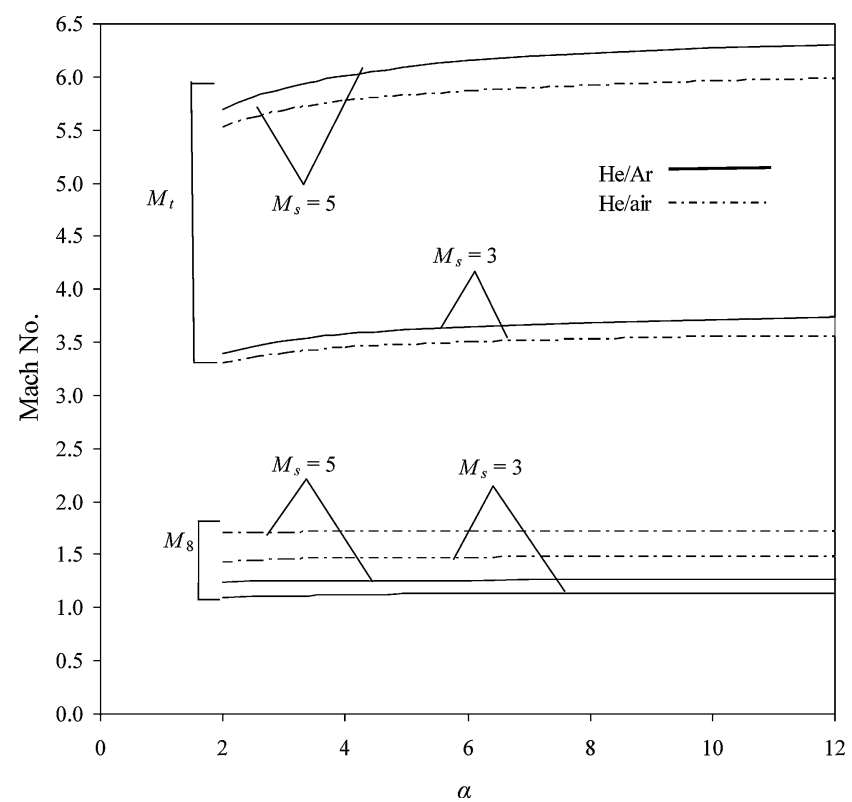

Fig. 2 Variation of $M_{t}$ and $M_{8}$ vs the contraction ratio $\alpha$.

\section{Regions 9 and 10}

Region 9 is downstream of the reflected shock when the end of the large-diameter tube is closed. Similarly, region 10 is downstream of the reflected shock in the small-diameter tube (Fig. 1). Equations for these two regions are given at the end of the Appendix.

\section{Results and Discussion}

A code was developed based on the foregoing model that is linear in that large loops are not required. The procedure in the code closely follows the order of presentation in the preceding section. Figure 2 shows $M_{t}$ and $M_{8}$ as functions of the contraction ratio when the high-/low-pressure gases are helium/air or helium/argon. The variation in $M_{t}$ and $M_{8}$ with $\alpha$ is slight when $\alpha$ exceeds about 5 . This asymptotic behavior holds for other parameters. Thus, an $\alpha$ value of 5 represents a large-area contraction ratio. Table 1 provides parametric results when $\gamma=1.4, \alpha=5$, and $M_{s}$ varies from 2.5 to 5.0. The expected increase in $M_{t}$ over $M_{s}$ and in $(p, T, w)_{8}$ over $(p, T, w)_{2}$ is evident. The percent increase, $\left(M_{t}-M_{s}\right) 10^{2} / M_{s}$, slowly increases from $15.64 \%$ at $M_{s}=2.5$ to $16.59 \%$ at $M_{s}=5.0$. Note that $M_{2}$ is transonic at low $M_{s}$ values, whereas $M_{8}$ is supersonic. Because $\alpha=5$ in Table $1, M_{5}$ equals 0.1167 for all $M_{s}$ values.

As already noted, the analysis is independent of the high-pressure gas. The last three columns in Table 1 show $p_{4} / p_{1}$ when the high-/ low-pressure gases are air/air, helium/air, and helium/argon, and $T_{4}=T_{1}$. As expected, when the high-pressure gas is air, the diaphragm pressure ratio becomes exorbitant when $M_{s}$ equals or exceeds 3.5. This is not the case, however, when helium is the highpressure gas. Note the lower $p_{4} / p_{1}$ pressure ratio for helium/argon compared to helium/air.

Figures 3 and 4 show $p_{10} / p_{9}$ and $T_{10} / T_{9}$, respectively, for the same high-/low-pressure gases as in Fig. 2. Recall that state 9 is for a conventional shock tube with $T_{1}=T_{4}$. Both the pressure and temperature are enhanced by the contraction, where the rate of in-

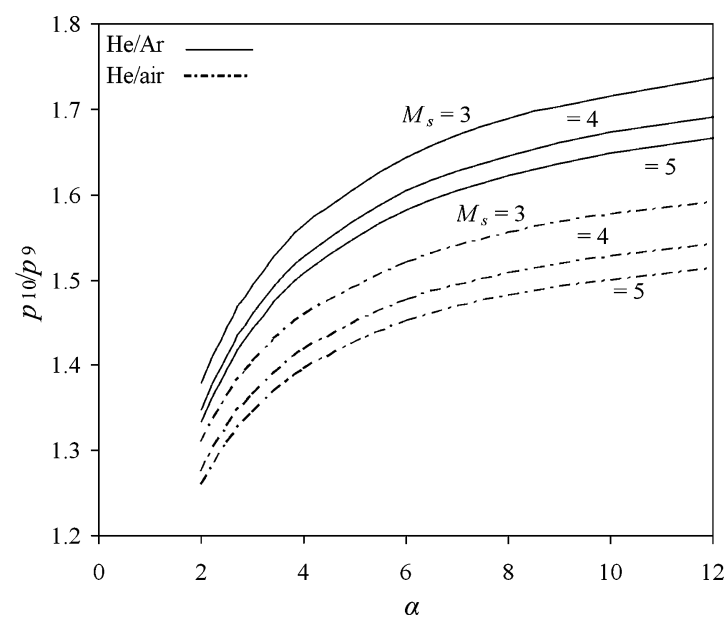

Fig. 3 Variation of $p_{10} / p_{9}$ vs the contraction ratio $\alpha$.

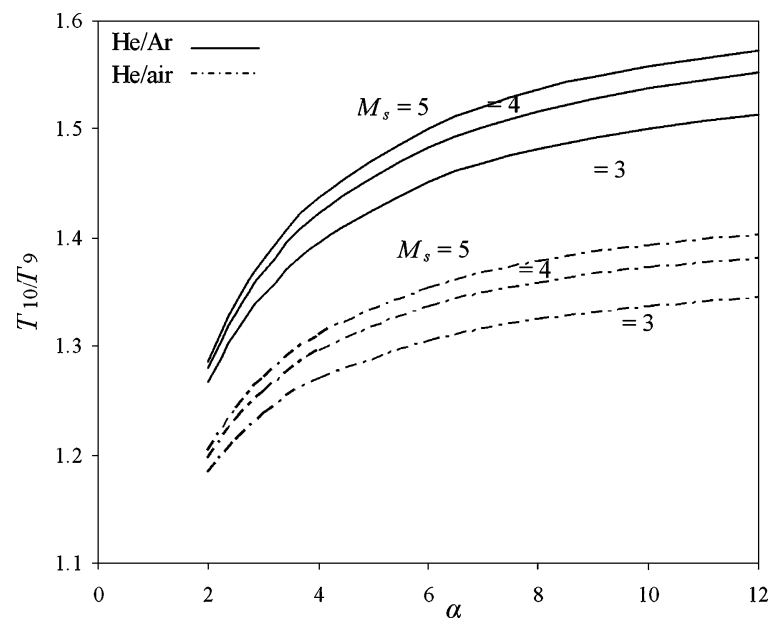

Fig. 4 Variation of $T_{10} / T_{9}$ vs the contraction ratio $\alpha$.

crease slows once $\alpha$ exceeds 5. For a given $\alpha, p_{10} / p_{9}$ and $T_{10} / T_{9}$ have opposite trends as $M_{s}$ increases. For $\alpha=5$, helium/argon, and $M_{s}=4, T_{10} / T_{9}$ is 1.46 . The $T_{10} / T_{9}$ parameter slowly increases with $\alpha$ and $M_{s}$. For $\alpha \gg 1$ and $M_{s} \gg 1$, however, it can reach a value as large as 1.6 . Hence, the increase in $T_{9}$, relative to $T_{10}$, is significantly larger than the $M_{t}$ increase, relative to $M_{s}$. A large $T_{10}$ temperature suggests the use of a contraction for radiative heat-transfer studies, high-temperature chemical kinetic and plasma studies, and for high-enthalpy shock-tunnel operation.

\section{Conclusions}

A simple, inviscid model is given for a shock tube with a large, abrupt contraction in the low-pressure side. Various limits and constraints are discussed. A unique, nondimensional solution only depends on $\gamma, M_{s}$, and the contraction ratio $\alpha$. A large $\alpha$ value is 5; above this value there is little change in shock-tube performance. Parametric calculations establish the performance improvement, both behind the transmitted shock and its end-wall reflection. 
The performance change stems from the increase in the transmitted shock Mach number relative to a tube without contraction. This performance improvement can be used in conjunction with other performance enhancement techniques.

\section{Appendix: Equations for States 2, 9, and 10}

The equations needed for state 2 are

$$
\begin{gathered}
p_{2} / p_{1}=[2 /(\gamma+1)] Y_{s} \\
T_{2} / T_{1}=[2 /(\gamma+1)]^{2}\left(X_{s} Y_{s} / M_{s}^{2}\right) \\
M_{2}=Z_{s} /\left(X_{s} Y_{s}\right)^{\frac{1}{2}} \\
R=\bar{R} / W \\
a_{1}=\left(\gamma R T_{1}\right)^{\frac{1}{2}} \\
w_{2} / a_{1}=[2 /(\gamma+1)]\left(Z_{s} / M_{s}\right) \\
w_{s} / a_{1}=M_{s} \\
a_{2} / a_{1}=[2 /(\gamma+1)]\left[\left(X_{s} Y_{s}\right)^{\frac{1}{2}} / M_{s}\right]
\end{gathered}
$$

where $X_{s}=X\left(M_{s}\right)$, and $w_{s}$ is the large-diameter tube's incident shock speed. The equations for the reflected shock regions, states 9 and 10 , are

$$
\begin{aligned}
& M_{r 9}=\left(Y_{s} / X_{s}\right)^{\frac{1}{2}} \\
& p_{9} / p_{1}=[2 /(\gamma+1)]\left(Y_{s} / X_{s}\right)\left\{[(3 \gamma-1) / 2] M_{s}^{2}-(\gamma-1)\right\} \\
& T_{9} / T_{1}=[2 /(\gamma+1)]^{2}\left(1 / M_{s}^{2}\right)\left[(\gamma-1) M_{s}^{2}+(3-\gamma) / 2\right] \\
& \quad \times\left\{[(3 \gamma-1) / 2] M_{s}^{2}-(\gamma-1)\right\} \\
& M_{r 10}=\left(Y_{t} / X_{t}\right)^{\frac{1}{2}} \\
& p_{10} / p_{1}=[2 /(\gamma+1)]\left(Y_{t} / X_{t}\right)\left\{[(3 \gamma-1) / 2] M_{t}^{2}-(\gamma-1)\right\} \\
& T_{10} / T_{1}=[2 /(\gamma+1)]^{2}\left(1 / M_{t}^{2}\right)\left[(\gamma-1) M_{t}^{2}+(3-\gamma) / 2\right] \\
& \quad \times\left\{[(3 \gamma-1) / 2] M_{t}^{2}-(\gamma-1)\right\}
\end{aligned}
$$

The $M_{r 9}$ and $M_{r 10}$ Mach numbers are for the respective reflected shock waves that measure the strength of the shocks, that is, they are in a shock-fixed frame.

\section{References}

${ }^{1}$ Schneider, S. P., and Haven, C. E., "Quiet-Flow Ludwieg Tube for High-Speed Transition Research," AIAA Journal, Vol. 33, No. 4, 1995, pp. 688-693.
${ }^{2}$ Verma, S. B., "Experimental Study of Flow Unsteadiness in a Mach 9 Compression Ramp Interaction Using a Laser Schlieren System," Measurement Science and Technology, Vol. 14, No. 7, 2003, pp. 989-997.

${ }^{3}$ Olivier, H., Jiang, Z., Yu, H., and Lu, F. K., "Detonation-Driven Shock Tubes and Tunnels," Advanced Hypersonic Test Facilities, edited by F. K. Lu and D. E. Marren, Vol. 198, AIAA, Reston, VA, 2002, pp. 135-203.

${ }^{4}$ Bogdanoff, D. W., "Improvement of Pump Tubes for Gas Guns and Shock Tube Drivers," AIAA Journal, Vol. 28, No. 3, 1990, pp. 483-491.

${ }^{5}$ Wilkins, M. E., and Carros, R. J., "Combustion Tests of OxygenHydrogen-Helium Mixtures at Loading Pressures up to 8000 Pounds per Square Inch,” NASA TN D-1892, Oct. 1963.

${ }^{6}$ Hiers, R. S., Loubsky, W. J., and Stewart, D. A., "Performance of a Combustion Driven Shock Tunnel with Application to the Tailored-Interface Operating Conditions," NASA TM X-54960, Dec. 1964.

${ }^{7}$ Itoh, K., Ueda, S., Komura, T., Sato, K., Takahashi, M., Miyajima, H., Tanno, H., and Muramoto, H., "Improvement of Free Piston Driver for High Enthalpy Shock Tunnel," Shock Waves, Vol. 8, No. 4, 1989, pp. 215-233.

${ }^{8}$ Sharma, S. P., and Park, C., "Operating Characteristics of a 60- and 10-cm Electric Arc Driven Shock Tube-Part I: The Driver," Journal of Thermophysics and Heat Transfer, Vol. 4, No. 3, 1990, pp. 259-265.

${ }^{9}$ Belanger, J., and Hornung, H. G., "A Combustion Driven Shock Tunnel to Complement the Free Piston Shock Tunnel T5 at GALCIT," AIAA Paper 92-3968, 1992.

${ }^{10}$ Bogdanoff, D. W., Zambrana, H. A., Cavolowsky, J. A., Newfield, M. E., Cornelison, C. J., and Miller, R. J., "Reactivation and Upgrade of the NASA Ames 16 Inch Shock Tunnel: Status Report," AIAA Paper 92-0327, 1992.

${ }^{11}$ Eitelberg, G., "First Results of Calibration and Use of the HEG," AIAA Paper 94-2525, 1994.

${ }^{12}$ Neely, A. J., and Morgan, R. G., "The Superorbital Expansion Tube Concept, Experiment and Analysis," Aeronautical Journal, Vol. 98, No. 973, 1994, pp. 97-105.

${ }^{13}$ Chue, R. S. M., Tsai, C. Y., Bakos, R. J., Erdos, J. I., and Rogers, R. C., "NASA's HYPULSE Facility at GASL-A Dual Mode, Dual Driver Reflected-Shock/Expansion Tunnel," Advanced Hypersonic Test Facilities, edited by F. K. Lu and D. E. Marren, Vol. 198, Progress in Astronautics and Aeronautics, AIAA, Reston, VA, 2002, pp. 29-71.

${ }^{14}$ Holden, M. S., and Parker, R. A., "LENS Hypervelocity Tunnels and Application to Vehicle Testing at Duplicated Flight Conditions," Advanced Hypersonic Test Facilities, edited by F. K. Lu and D. E. Marren, Vol. 198, Progress in Astronautics and Aeronautics, AIAA, Reston, VA, 2002, pp. 73-110.

${ }^{15}$ Harwell, K. E., and Jahn, R. G., "Initial Ionization Rates in ShockHeated Argon, Krypton, and Xenon," Physics of Fluids, Vol. 7, No. 2, 1964, pp. 214-222.

${ }^{16}$ Johnson, J. A., III, Lin, I., and Santiago, J. P., "Turbulent Collisional Ionising Shock Waves in Argon," Journal of Physics D: Applied Physics, Vol. 23, No. 3, 1990, pp. 662-672.

${ }^{17}$ Bogdanoff, D. W., and Park, C., "Radiative Interaction Between Driver and Driven Gases in an Arc-Driven Shock-Tube," Shock Waves, Vol. 12, No. 3, 2002, pp. 205-214

${ }^{18}$ Alpher, R. A., and White, D. R., "Flow in Shock Tubes with Area Change at the Diaphragm Section," Journal of Fluid Mechanics, Vol. 3, 1958, pp. 457-470.

${ }^{19}$ Salas, M. D., "Shock Wave Interaction with an Abrupt Area Change," NASA TP 3113, Aug. 1991.

${ }^{20}$ Emanuel, G., Gasdynamics: Theory and Applications, AIAA, New York, 1986.

${ }^{21}$ Emanuel, G., Analytical Fluid Dynamics, 2nd ed., CRC Press, Boca Raton, FL, 2001, Secs. 12.4 and 12.5.

M. Sichel Associate Editor 